\title{
Exact Ground States of One-Dimensional Quantum Systems: Matrix Product Approach
}

\author{
Gang $\mathrm{Su}^{*}$ \\ Institut für Theoretische Physik, Universität zu Köln \\ Zülpicher Strasse 77, D-50937 Köln, Germany
}

\begin{abstract}
By using the so-called matrix-product ground state approach, a few onedimensional quantum systems, including a frustrated spin-1/2 Heisenberg ladder, the ferromagnetic t-J-V model at half-filling, the antiferromagnetic $J_{z}-V$ at $2 / 3$ filling and the antiferromagnetic $t-J_{z}-V$ model at halffilling, are solved exactly. The correlation functions in the ground states are calculated respectively. Some relevant results are also discussed.
\end{abstract}

PACS numbers: 75.10.Jm, 71.10+x, 05.50

Typeset using REVTEX 


\section{INTRODUCTION}

Recently there has been growing interest on one-dimensional (1D) quantum systems due to a variety of reasons. Since the difficulty in dealing with many-body problems the methods in obtaining exact results in $1 \mathrm{D}$ are rare. As is well-known, a few $1 \mathrm{D}$ many-body problems can be exactly solved by the Bethe ansatz, as the systems satisfy the so-called Yang-Baxter equation [1]. Actually, Bethe ansatz is a powerful and efficient method in obtaining exact results including ground state and excited states as well as thermodynamics. However, a lot of 1D quantum systems does not obey Yang-Baxter equation, and thus are non-integrable. In this situation, it is difficult, due to absence of a systematic method, to obtain some exact results of 1D many-body systems. Amongst very few existing exact methods there is a so-called matrix product (MP) ground state approach which can be applied to obtain exact ground-state properties of 1D quantum systems. This method was established a few years ago by Klümper et al [2] where they studied the exact ground state of a large class of antiferromagnetic spin-1 chains. In those cases the method was really efficient. The basic idea of the method is that the global ground state can be constructed in the form of a matrix product of single-site states. This state is usually said optimal in the sense that it is the product of all the local ground states of the local interaction $h_{j, j+1}$ (see below). To show that the MP state is really the ground state, one can diagonalize $h_{j, j+1}$ exactly in all local eigenstates. If the eigenvalue of $h_{j, j+1}$ in the MP ground state is not larger than the minimum of all eigenvalues of $h_{j, j+1}$ diagonalized in all local eigenstates, then the MP state is the global ground state of the whole Hamiltonian. The similar spirit has been applied to construct the exact ground states of generalized Hubbard models [3]. In this paper, we will apply the MP approach to a few quantum systems including a frustrated spin-1/2 Heisenberg spin ladder, and several simplified cases of t-J-V model. The correlation functions of the ground states are obtained. Some relevant results are also addressed. 


\section{A FRUSTRATED SPIN-1/2 HEISENBERG LADDER}

Investigations on spin ladders have attracted a lot of attention in recent years [4 [12. This is usually motivated from the following possible reasons: one is that the Heisenberg spin ladder (two antiferromagnetically coupled Heisenberg antiferromagnetic spin-1/2 chains or two ferromagnetically coupled such chains [13, 14]) provides a simple model to gain insight into the underlying physics of crossover from 1D chain to 2D square lattice; one is due to the Haldane's conjecture [15] - which states that a quantum antiferromagnet with integral spin has different properties from one with half-integral spin, i.e., the former has a unique ground state, a gap to the excited state and exponential decaying ground-state correlation functions, which have been confirmed in experiments, while the latter does not - people believe that the Heisenberg spin ladder is probably in the same phase as the Heisenberg spin-1 antiferromagnetic chain [9,10,12]; and the other is that spin ladders are intimately related to magnetic properties of some realistic materials (for example, $S r_{n-1} C u_{n+1} \mathrm{O}_{2 n}$ [16,8] and $(\mathrm{VO})_{2} P_{2} \mathrm{O}_{7}$ [17]). Besides, frustrated spin ladders are also interesting, because they are believed to be responsible for physical properties of stoichiometric $S r_{n-1} C u_{n+1} O_{2 n}$ compounds $[8]$. Exact solutions on spin ladders are rare so far, whereas earlier studies on these models are either approximate (mean-field treatment or bosonization) or numerical, although some consensus is qualitatively made. Any exact result on these models will hence be necessary. In this section, we report that a frustrated spin-1/2 Heisenberg ladder, with properly constricted parameters, can be solved exactly by using the MP ground state approach.

We consider a spin-1/2 Heisenberg ladder with diagonal coupling, described by the following Hamiltonian

$$
\begin{aligned}
H & =J_{1} \sum_{n=1,2} \sum_{j=1}^{L} \mathbf{S}_{n}(j) \cdot \mathbf{S}_{n}(j+1)+J_{2} \sum_{j=1}^{L} \mathbf{S}_{1}(j) \cdot \mathbf{S}_{2}(j) \\
& +J_{3} \sum_{j=1}^{L}\left[\mathbf{S}_{1}(j) \cdot \mathbf{S}_{2}(j+1)+\mathbf{S}_{2}(j) \cdot \mathbf{S}_{1}(j+1)\right]
\end{aligned}
$$




$$
+\frac{2\left(J_{1}+J_{3}\right)+J_{2}}{4}
$$

where $j$ runs over all rungs and $n$ over two legs: 1 and $2, L$ is the length of the ladder, $\mathbf{S}_{n}(j)$ is spin-1/2 operator on the rung $j$ along leg $n$ of the ladder, $J_{1}$ is the interaction along the legs, $J_{2}$ is the coupling along the rungs, and $J_{3}$ is the diagonal coupling between the two nearest neighbor rungs. A constant (the last term of (1)) is added for convenience of our purpose. The periodic boundary condition is assumed along the ladder. To guarantee the solvability of the ladder, we have to confine the parameters to satisfy the following conditions:

$$
0<J_{1} \leq-\frac{J_{2} J_{3}}{J_{2}+2 J_{3}}, \quad J_{2}<0, \quad J_{3}<0
$$

Under the conditions of (2), one may see that spins on the ladder are frustratedly distributed along the legs and rungs, minimizing the total energy of the system. We mean the frustration in the present model just in this sense. In the following we for brevity denote the local eigenstates $\left|+\frac{1}{2}\right\rangle_{j}$ and $\left|-\frac{1}{2}\right\rangle_{j}$ of $S^{z}(j)$ simply by $|+\rangle_{j}$ and $|-\rangle_{j}$, respectively. We denote a local state on each rung by $|a\rangle_{j}^{m}|b\rangle_{j}^{n}$, where $\{a, b\}=\{+,-\}$, and $\{m, n\}=\{1,2\}$ identifying the legs. Introduce a permutation operator

$$
P_{j, l}^{(m, n)}=\frac{1}{2}\left[1+4 \mathbf{S}_{m}(j) \cdot \mathbf{S}_{n}(l)\right]
$$

The operator has the property:

$$
P_{j, l}^{(m, n)}|a\rangle_{j}^{m}|b\rangle_{l}^{n}=|b\rangle_{j}^{m}|a\rangle_{l}^{n}
$$

With the aid of (3), we rewrite (1) as

$$
H=\sum_{j=1}^{L} h_{j, j+1},
$$

with the local interaction

$$
h_{j, j+1}=\frac{J_{1}}{2}\left(P_{j, j+1}^{(1,1)}+P_{j, j+1}^{(2,2)}\right)+\frac{J_{2}}{4}\left(P_{j, j}^{(1,2)}+P_{j+1, j+1}^{(1,2)}\right)+\frac{J_{3}}{2}\left(P_{j, j+1}^{(1,2)}+P_{j, j+1}^{(2,1)}\right),
$$

where we have made use of the periodic boundary condition along the ladder. 
Following the discussions in Ref. [2], and using the local eigenstates $|+\rangle_{j}$ and $|-\rangle_{j}$ of $S_{j}^{z}$, we define a local $2 \times 2$ matrix on each rung by

$$
g_{j}=\left(\begin{array}{c}
|+\rangle_{j}^{1}|-\rangle_{j}^{2}+|-\rangle_{j}^{1}|+\rangle_{j}^{2}|+\rangle_{j}^{1}|+\rangle_{j}^{2}+|-\rangle_{j}^{1}|-\rangle_{j}^{2} \\
|+\rangle_{j}^{1}|+\rangle_{j}^{2}+|-\rangle_{j}^{1}|-\rangle_{j}^{2}|+\rangle_{j}^{1}|-\rangle_{j}^{2}+|-\rangle_{j}^{1}|+\rangle_{j}^{2}
\end{array}\right)
$$

and a global state

$$
\left|\Psi_{0}\right\rangle=\operatorname{Tr}_{1} \otimes g_{2} \otimes \cdots \otimes g_{L}
$$

where $\otimes$ denotes usual matrix multiplication of $2 \times 2$ matrices with a tensor product of the matrix elements. It can be found that

$$
\begin{array}{r}
H\left|\Psi_{0}\right\rangle=E_{0}\left|\Psi_{0}\right\rangle, \\
E_{0}=\left(J_{1}+\frac{J_{2}}{2}+J_{3}\right) L .
\end{array}
$$

As we have the constraint (2), thus $E_{0}<0$. Now let us show $\left|\Psi_{0}\right\rangle$ is a ground state of $H$. To realize this purpose, we in turn need to prove $g_{j} \otimes g_{j+1}$ and thus $\left|\Psi_{0}\right\rangle$ to be the ground state of $h_{j, j+1}$. After a little tedious algebraic calculations, one may find that

$$
h_{j, j+1}\left(g_{j} \otimes g_{j+1}\right)=\left(J_{1}+\frac{J_{2}}{2}+J_{3}\right)\left(g_{j} \otimes g_{j+1}\right),
$$

namely, $g_{j} \otimes g_{j+1}$ is an eigenstate of $h_{j, j+1}$ with eigenvalue $E_{0} / L$. On the other hand, $h_{j, j+1}$ can be exactly diagonalized in all possible 16 eigenstates on a plaquette consisting of two rungs $(j$ and $j+1)$ and two corresponding legs. It is not hard to obtain the eigenvalues as follows: $J_{1}$ (3-fold), $\frac{J_{2}}{2}$ (3-fold), $J_{3}$ (3-fold), $J_{1}+\frac{J_{2}}{2}+J_{3}$ (5-fold), $\pm\left[J_{1}^{2}-\frac{1}{2} J_{1} J_{2}+\frac{1}{4} J_{2}^{2}-\right.$ $\left.J_{1} J_{3}-\frac{1}{2} J_{2} J_{3}+J_{3}^{2}\right]^{\frac{1}{2}}$. One can easily check that $E_{0} / L=J_{1}+\frac{J_{2}}{2}+J_{3}$ is the lowest eigenvalue between them, under the conditions of (2). Thus, $g_{j} \otimes g_{j+1}$ is really the ground state of $h_{j, j+1}$, and therefore $\left|\Psi_{0}\right\rangle$ is also the ground state of $h_{j, j+1}$, which in turn proves that $\left|\Psi_{0}\right\rangle$ is the global ground state of $H$, and $E_{0}$ is the ground-state energy.

Similar to the calculations in Ref. [2], we can obtain correlation functions in $\left|\Psi_{0}\right\rangle$ state by using the transfer matrix method. The norm $\left\langle\Psi_{0} \mid \Psi_{0}\right\rangle$ can be obtained by 


$$
\left\langle\Psi_{0} \mid \Psi_{0}\right\rangle=\sum_{\left\{n_{\alpha}, m_{\alpha}\right\}} g_{n_{1} n_{2}}^{+} g_{n_{2} n_{3}}^{+} \cdots g_{n_{L} n_{1}}^{+} g_{m_{1} m_{2}} g_{m_{2} m_{3}} \cdots g_{m_{L} m_{1}}=\operatorname{Tr} G^{L}
$$

where $G$ is the transfer matrix, with elements $G_{\mu_{1} \mu_{2}} \equiv G_{\left(n_{1} m_{1}\right),\left(n_{2} m_{2}\right)}=g_{n_{1} n_{2}}^{+} g_{m_{1} m_{2}}$ where the ordering of multi-indices is chosen as usual: $\mu=1,2,3,4 \leftrightarrow(11),(12),(21),(22)$. The $4 \times 4$ transfer matrix has four eigenvalues: 0 (2-fold) and 4 (2-fold). Thus, one can write $G\left|e_{n}\right\rangle=\lambda_{n}\left|e_{n}\right\rangle$, with $\lambda_{n}(n=1,2,3,4)$ the eigenvalues $\left(\lambda_{1,2}=0, \lambda_{3,4}=4\right)$, and $\left|e_{n}\right\rangle$ the corresponding normalized eigenvectors which have alternative forms

$$
\left|e_{1}\right\rangle=\left(\begin{array}{c}
\frac{1}{2} \\
\frac{1}{2} \\
-\frac{1}{2} \\
-\frac{1}{2}
\end{array}\right), \quad\left|e_{2}\right\rangle=\left(\begin{array}{c}
-\frac{1}{2} \\
\frac{1}{2} \\
-\frac{1}{2} \\
\frac{1}{2}
\end{array}\right), \quad\left|e_{3}\right\rangle=\left(\begin{array}{c}
\frac{1}{2} \\
\frac{1}{2} \\
\frac{1}{2} \\
\frac{1}{2}
\end{array}\right), \quad\left|e_{4}\right\rangle=\left(\begin{array}{c}
-\frac{1}{2} \\
\frac{1}{2} \\
\frac{1}{2} \\
-\frac{1}{2}
\end{array}\right) .
$$

One may verify that $\left\{\left|e_{n}\right\rangle\right\}$ forms a complete set, which can be used to calculate the trace in the following. Evidently, $\left\langle\Psi_{0} \mid \Psi_{0}\right\rangle=2 \cdot 4^{L}$. The one-site expectations can be evaluated by

$$
\langle A\rangle=\frac{\left\langle\Psi_{0}|A| \Psi_{0}\right\rangle}{\left\langle\Psi_{0} \mid \Psi_{0}\right\rangle}=\left(\operatorname{Tr} G^{L}\right)^{-1} \operatorname{Tr} Z(A) G^{L-1},
$$

with $Z(A)_{\mu_{1} \mu_{2}} \equiv g_{n_{1} n_{2}}^{+} A g_{m_{1} m_{2}}$. The 2-site correlations of operators $A(1)$ at site 1 and $B(r)$ at site $r$ can be calculated by

$$
\langle A(1) B(r)\rangle=\left(\operatorname{Tr} G^{L}\right)^{-1} \operatorname{Tr} Z(A) G^{r-2} Z(B) G^{L-r} .
$$

In accordance with (12)-(14), we can obtain the following expectations:

$$
\begin{aligned}
& \left\langle S_{1,2}^{z}(j)\right\rangle=0, \quad\left\langle S_{t o t}^{z}\right\rangle=\sum_{j=1}^{L}\left\langle S_{1}^{z}(j)+S_{2}^{z}(j)\right\rangle=0, \quad\left\langle S_{1,2}^{+}(j)\right\rangle=0, \\
& \left\langle S_{1,2}^{z}(1) S_{1,2}^{z}(r)\right\rangle=\left\langle S_{1,2}^{z}(1) S_{2,1}^{z}(r)\right\rangle=0, \\
& \left\langle\left(S_{1,2}^{z}(j)\right)^{2}\right\rangle=\left\langle S_{1,2}^{+}(1) S_{1,2}^{-}(r)\right\rangle=\left\langle S_{1,2}^{+}(1) S_{2,1}^{-}(r)\right\rangle=\frac{1}{4},
\end{aligned}
$$

for $r \geq 2$, where the technical details refer to Ref. [2]. We observe that in the ground state the model has transverse fluctuations for the transverse correlation functions are uniform with varying spatial distances, implying the correlation length for transverse magnetic orderings 
is infinite, while it does not exhibit longitudinal magnetic order and fluctuations. As can be seen, $\left|\Psi_{0}\right\rangle$ is XY magnetic ordered.

To identify the structure of the state, one can use a matrix $u=\left(\begin{array}{cc}1 & 1 \\ 1 & -1\end{array}\right)$ to make a similarity transformation on $g_{j}$ as $g_{j}^{\prime}=u g_{j} u^{-1}=\left(\begin{array}{cc}a+b & 0 \\ 0 & a-b\end{array}\right)$ with $a=|+\rangle_{j}^{1}|-\rangle_{j}^{2}+$ $|-\rangle_{j}^{1}|+\rangle_{j}^{2}$ and $b=|+\rangle_{j}^{1}|+\rangle_{j}^{2}+|-\rangle_{j}^{1}|-\rangle_{j}^{2}$. Thus $\left|\Psi_{0}\right\rangle=\operatorname{Trg}_{1}^{\prime} \otimes g_{2}^{\prime} \otimes \cdots \otimes g_{L}^{\prime}=|a+b\rangle_{1} \mid a+$ $b\rangle_{2} \cdots|a+b\rangle_{L}+|a-b\rangle_{1}|a-b\rangle_{2} \cdots|a-b\rangle_{L}$. Obviously, the global state is a linear combination of a direct product of all possible spin configurations on each rung, and thus has transverse fluctuations but without longitudinal ones, as revealed by the expectations. In addition, since $g_{j}$ is a mixture of singlet and triplet states, the excitation from the ground state $\left|\Psi_{0}\right\rangle$ may be gapless.

A simple argument can show that the ground state is degenerate. For instance, if one replaces the four entries of matrix $g_{j}$ in (7) by the same expression: $|+\rangle_{j}^{1}|+\rangle_{j}^{2}$ or $|-\rangle_{j}^{1}|-\rangle_{j}^{2}$, one will find that the replaced MPG state has the same eigenvalue as that of $\left|\Psi_{0}\right\rangle$. Actually, such states are nothing but the fully polarized ferromagnetic states, which can be implemented through a similarity matrix transformation on $g_{j}$. One can also compute expectations of some physical quantities in the polarized ferromagnetic state, and will find that they have different values as in $\left|\Psi_{0}\right\rangle$ state, while the longitudinal correlation functions are still uniform in spatial space. Hence $\left|\Psi_{0}\right\rangle$ and the fully polarized ferromagnetic state are not the same, suggesting that the ground state is not unique.

That the model has the fully polarized ferromagnetic ground state is not surprising, because the coupling along the rungs $\left(J_{2}<0\right)$ and the diagonal coupling between the rungs $\left(J_{3}<0\right)$ favour to form triplets and thus prefer to form ferromagnetic order, while the strength along the legs, though it is positive and favours to form antiferromagnetic order, is smaller than the other two interactions. The competing result would lead to favour ferromagnetism. The present result just confirms the intuitive argument. However, the XY magnetic ordered ground state exist in the model is yet plausible in physics. Though 
the spins on each rung of the ladder prefer to form triplets, minimizing the energy of the system, the alignment of the spins along positive direction has the equal weight as that along negative direction, and thus the resultant state may have transverse magnetic fluctuations but without longitudinal magnetic order, as can be seen clearly from the explicit form of $\left|\Psi_{0}\right\rangle$. As the ground state is degenerate, one may anticipate that the spin rotational symmetry is spontaneously broken in the ground state, as it should be. To this end, one may note that the advantage of MP ground state approach applying to this model lies in having found a ground state with transverse magnetic fluctuations, thereby showing the ground state of the model is not unique, and the excitation is gapless.

To conclude this section, the frustrated spin-1/2 Heisenberg ladder, defined in (1), is solved exactly by using the MP ground state approach. It is shown that the ground state of the model is degenerate, as a magnetic ordered state with transverse fluctuations and a fully polarized ferromagnetic state coexist in the ground state. The excitation from the ground state may be gapless. The correlation functions are uniform in spatial space, implying that the correlation length is infinite, and thus the system is critical in the ground state. One may observe that this model thus provides an example that quasi one-dimensional frustrated Heisenberg model can exhibit magnetic order in the ground state, although the MerminWagner thermodynamic fluctuations [18] enable the system probably not to possess such an order at finite temperatures. We expect that the present result, on the one hand, may shed some light on the theoretical basis of some quasi one-dimensional ferromagnets, and on the other hand, may provide a clue to experimentalists to synthesize new quasi one-dimensional ferromagnetic materials, particularly organic magnets.

\section{T-J-V MODEL}

This model, as an extension of the usual t-J model, first proposed by Schlottman [19 for heavy-fermions, and later studied numerically by others [20 22]. Apart from the supersymmetric points [19], this model is non-integrable. The system is described by the following 
Hamiltonian

$$
H_{t J V}=\sum_{j=1}^{L} h_{j, j+1}
$$

with the local interaction

$$
h_{j, j+1}=-t \sum_{\sigma}\left(c_{j, \sigma}^{\dagger} c_{j+1, \sigma}+h . c .\right)+\frac{J}{2}\left(S_{j}^{+} S_{j+1}^{-}+S_{j}^{-} S_{j+1}^{+}\right)+J_{z} S_{j}^{z} S_{j+1}^{z}+V n_{j} n_{j+1},
$$

on $1 \mathrm{D}$ chain with the length $L$ even, where $c_{j, \sigma}$ is the annihilation operator for an electron with spin $\sigma(=\uparrow, \downarrow)$ at site $j,\left\{S_{j}\right\}$ are spin-1/2 operators, $n_{j}=\sum_{\sigma} c_{j, \sigma}^{\dagger} c_{j, \sigma}$ is the electron number operator at site $j, t$ is the hopping matrix element, $J, J_{z}$ are anisotropic spinexchange interactions, and $V$ is the strength of density-density interaction. The periodic boundary conditions are assumed. We further suppose that, as usual, the double-occupancy of every site is forbidden due to the existence of a large on-site Coulomb repulsion, i.e., each site has either one electron (with spin up or down) or empty. Therefore, there are three possible electronic states at a given site $j:|0\rangle$, the Fock vacuum satisfying $c_{j, \sigma}|0\rangle \equiv 0$; $|\uparrow\rangle \equiv c_{j, \uparrow}^{\dagger}|0\rangle$; and $|\downarrow\rangle \equiv c_{j, \downarrow}^{\dagger}|0\rangle$. Since there exists such a property, the problem, as first noted by Schlottman [19] in the t-J-V model, can be rewritten in terms of spin operators corresponding to $S=1$ in the restricted Hilbert space, which implies that the model should have similar behaviors as that of $S=1$ spin chain. This defines the t-J-V model. Since it is non-integrable off supersymmetric points, it is difficult to obtain the exact results of this model. In the following we will concentrate on a few special cases, which can be solved exactly using MP approach.

\section{A. Ferromagnetic t-J-V model at half-filling}

In this case we assume that $J_{z}=J<0$ and $t \geq 0$. Similar to Sec.II, we define a $2 \times 2$ matrix

$$
g_{j}^{\prime}=\left(\begin{array}{cc}
|\uparrow\rangle+|\downarrow\rangle & \frac{1}{\sqrt{a}}(|\uparrow\rangle-|\downarrow\rangle) \\
\frac{1}{\sqrt{a}}(|\downarrow\rangle-|\uparrow\rangle) & |\downarrow\rangle+|\uparrow\rangle
\end{array}\right)_{j}
$$


with $a>0$ a parameter, and propose an ansatz for the global ground state

$$
\left|\Phi_{1}\right\rangle=\operatorname{Tr}_{1}^{\prime} \otimes g_{2}^{\prime} \otimes \cdots \otimes g_{L}^{\prime},
$$

where the notation is the same as in Sec.II. Following exactly the analyses in Sec.II, it is readily show that $\left|\Phi_{1}\right\rangle$ is the global ground state, with eigenvalue $\left(V+\frac{J}{4}\right) L$, of the ferromagnetic t-J-V model under the following constraint:

$$
t \geq 0, \quad J<0, \quad V \leq-t-\frac{J}{4} .
$$

The expectation values in the ground state can be calculated directly using the transfer matrix method. Though the finite-size results are available, we are only interested in those in the thermodynamic limit. Henceafter we mean the result just for $L \rightarrow \infty$. It can be found that

$$
\left\langle S_{j}^{z}\right\rangle=0, \quad\left\langle S_{\text {tot }}^{z}\right\rangle=\left\langle\sum_{j=1}^{L} S_{j}^{z}\right\rangle=0, \quad\left\langle S_{j}^{ \pm}\right\rangle=\frac{1}{2} \frac{a-1}{a+1}, \quad\left\langle n_{j}\right\rangle=1, \quad\left\langle\left(S_{j}^{z}\right)^{2}\right\rangle=\frac{1}{4}, \quad\left\langle J_{j}\right\rangle=0,
$$

with $J_{j}=i t \sum_{\sigma}\left(c_{j+1, \sigma}^{\dagger} c_{j, \sigma}-c_{j, \sigma}^{\dagger} c_{j+1, \sigma}\right)$, the current density. Therefore, we see that the ground state has XY ordering and is insulating. The two-point correlation functions are evaluated

$$
\left\langle S_{1}^{z} S_{r}^{z}\right\rangle=0,\left\langle S_{1}^{+} S_{r}^{-}\right\rangle=\left\langle S_{1}^{-} S_{r}^{+}\right\rangle=\frac{1}{4},\left\langle n_{1} n_{r}\right\rangle=1,\left\langle n_{1} S_{r}^{z}\right\rangle=0 .
$$

When $a=1,\left\langle S_{j}^{ \pm}\right\rangle=0$, implying that there is a phase transition from one XY ordered state with nonvanishing order parameter into another $\mathrm{XY}$ ordered state in which the order parameter vanishes while the transverse correlation function is uniform. Since the correlation length of transverse correlation functions is infinite, the system is critical in the ground state. An interesting observation is that, the state is still the ground state, if $J=0$ so long as $V \leq-t$. This fact suggests that the density-density interaction seems to play a significant role in the mechanism of the origin of ferromagnetism. Similar to arguments in Sec.II, the ground state of the system may be degenerate with the fully polarized ferromagnetic state. Since the symmetry breaking requires the degeneracy of the ground states, the present result is reasonable, as (23) shows that the spin rotational symmetry is spontaneously broken in the ground state. If one makes a particle-hole transformation in the system, then he will find the transformed system will exhibit off-diagonal long-range order in the ground state. 


\section{B. Antiferromagnetic $J_{z}-V$ model at $2 / 3$ filling}

Define a local $2 \times 2$ matrix

$$
g_{j}^{\prime \prime}=\left(\begin{array}{cc}
|\uparrow\rangle+|\downarrow\rangle+|0\rangle & |\uparrow\rangle-|\downarrow\rangle-|0\rangle \\
|\downarrow\rangle-|\uparrow\rangle+|0\rangle & -|\downarrow\rangle-|\uparrow\rangle-|0\rangle
\end{array}\right)_{j}
$$

and an ansatz for the global ground state of $H_{t J V}$

$$
\left|\Phi_{2}\right\rangle=\operatorname{Tr}_{1}^{\prime \prime} \otimes g_{2}^{\prime \prime} \otimes \cdots \otimes g_{L}^{\prime \prime}
$$

To make $\left|\Phi_{2}\right\rangle$ be the global ground state of $H_{t J V}$, tracing the exact way in Sec.II, the following conditions must be satisfied:

$$
t=J=0, \quad V=\frac{J_{z}}{4}, \quad J_{z}>0 .
$$

One can see that, $\left|\Phi_{2}\right\rangle$ is actually the global ground state, with eigenvalue 0 , of antiferromagnetic Ising model with density-density interaction described by the following Hamiltonian

$$
H_{J_{z} V}=J_{z} \sum_{j=1}^{L} S_{j}^{z} S_{j+1}^{z}+\frac{J_{z}}{4} \sum_{j=1}^{L} n_{j} n_{j+1} .
$$

It is well-known that this model without the density-density interaction has been solved by Ising [23] seventy years ago. Now we have applied matrix product to construct the global ground state of the Ising model with density-density interaction. Applying the similar arguments in Ref. [2] one can show that the ground state is unique. One will see later that the state is at $2 / 3$ filling. The state is thus nontrivial and is optimal.

The expectation values in the ground state can be obtained:

$$
\left\langle S_{j}^{z}\right\rangle=0, \quad\left\langle S_{\text {tot }}^{z}\right\rangle=\left\langle\sum_{j=1}^{L} S_{j}^{z}\right\rangle=0, \quad\left\langle S_{j}^{ \pm}\right\rangle=0, \quad\left\langle n_{j}\right\rangle=\frac{2}{3}, \quad\left\langle\left(S_{j}^{z}\right)^{2}\right\rangle=\frac{1}{6} .
$$

The spin-spin correlation function is nontrivial

$$
\left\langle S_{1}^{z} S_{r}^{z}\right\rangle=0.0331456-0.0625(-1)^{r}, \quad(r \geq 2)
$$

which means that the global ground state is antiferromagnetic, and has long-range order. Since the system is localized, the ground state should be insulating. The spin-charge correlation function is 


$$
\left\langle S_{1}^{z} n_{r}\right\rangle=0.0331456-0.04167(-1)^{r}, \quad(r \geq 2)
$$

which suggests that the spin and charge are not separated in the ground state. The other correlation functions are:

$$
\left\langle S_{1}^{+} S_{r}^{-}\right\rangle=\left\langle S_{1}^{-} S_{r}^{+}\right\rangle=0, \quad\left\langle n_{1} n_{r}\right\rangle=0.5, \quad\left\langle\left(S_{1}^{z}\right)^{2}\left(S_{r}^{z}\right)^{2}\right\rangle=0.03125 .
$$

These correlation functions are nontrivial, and it seems that they appear for the first time. One may note that these properties are quite different from those of Ising model [1,23].

\section{Antiferromagnetic $t-J_{z}-V$ model at half-filling}

Consider the simplified version of $H_{t J V}$, described by the Hamiltonian (18) with $J=0$, which we call the $t-J_{z}-V$ model. Below we will show that the ground states of this simplied model can be constructed using MP ground state approach at half-filling. As before, we define an $2 \times 2$ matrix

$$
g_{j}^{\prime \prime \prime}=\left(\begin{array}{cc}
|\uparrow\rangle+|\downarrow\rangle & |\uparrow\rangle-|\downarrow\rangle \\
|\downarrow\rangle-|\uparrow\rangle & -|\downarrow\rangle-|\uparrow\rangle
\end{array}\right)_{j} .
$$

We propose an ansatz

$$
\left|\Phi_{3}\right\rangle=\operatorname{Tr}_{1}^{\prime \prime \prime} \otimes g_{2}^{\prime \prime \prime} \otimes \cdots \otimes g_{L}^{\prime \prime \prime}
$$

Pursuing the same procedure as in Sec.II, one can find that $\left|\Phi_{3}\right\rangle$ is really the global ground state, with eigenvalue $\left(V-\frac{J_{z}}{4}\right) L$, of the $t-J_{z}-V$ model, subject to the following constraint

$$
J_{z}>0, \quad t>0, \quad V \leq-t+\frac{J_{z}}{4} .
$$

Similar to the arguments in Ref. [2], it can be shown that the global ground state is unique. The expectation values in the ground state are obtained

$$
\left\langle S_{j}^{z}\right\rangle=0, \quad\left\langle S_{\text {tot }}^{z}\right\rangle=\left\langle\sum_{j=1}^{L} S_{j}^{z}\right\rangle=0, \quad\left\langle S_{j}^{ \pm}\right\rangle=0, \quad\left\langle n_{j}\right\rangle=1, \quad\left\langle\left(S_{j}^{z}\right)^{2}\right\rangle=\frac{1}{4} .
$$


The global ground state is thus antiferromagnetic. The spin-spin correlation function is found to be

$$
\left\langle S_{1}^{z} S_{r}^{z}\right\rangle=-\frac{1}{4}(-1)^{r}, \quad(r \geq 2)
$$

which is nothing but the result of Ising model [1,23]. This fact shows that the antiferromagnetic $t-J_{z}-V$ model at half-filling has the same properties as the Ising model, implying that the model at half-filling can be mapped to the Ising model. Actually, this is true, because the hopping term, at half-filling, plays no role in the restricted Hilbert space without double-occupied sites. The other two-point correlation functions are

$$
\left\langle S_{1}^{+} S_{r}^{-}\right\rangle=\left\langle S_{1}^{-} S_{r}^{+}\right\rangle=0, \quad\left\langle n_{1} n_{r}\right\rangle=1, \quad\left\langle S_{1}^{z} n_{r}\right\rangle=0, \quad\left\langle\left(S_{1}^{z}\right)^{2}\left(S_{r}^{z}\right)^{2}\right\rangle=0.03125
$$

One may observe that the behaviors of correlators at half-filling are quite different from those at $2 / 3$ filling, as discussed in last subsection.

\section{SUMMARY AND CONCLUSION}

We have studied the exact ground-state properties of a few 1D quantum systems including a frustrated spin-1/2 Heisenberg ladder, a ferromagnetic t-J-V model, an antiferromagnetic $J_{z}-V$ model at $2 / 3$ filling and an antiferromagnetic $t-J_{z}-V$ model at half-filling, by using the matrix product ground state approach. The correlation functions were obtained in the ground states.

It is shown that the ground state of the frustrated spin-1/2 Heisenberg ladder with restricted parameters is magnetic ordered and is degenerate. The excitation from the ground state may be gapless. The correlation functions are uniform in spatial space, implying that the system is critical in the ground state in the sense that the correlation length is infinite.

The exact ground state of the ferromagnetic t-J-V model with proper parameters was constructed at half-filling. It is found that the ground state has magnetic long-range order,

and is also degenerate. A phase transition is found in the system. The spin rotational 
symmetry is spontaneously broken in the state. The correlation function is uniform, implies that the system is also critical in the ground state.

The global ground state of the antiferromagnetic $J_{z}-V$ model with $V=J_{z} / 4$ is found to be antiferromagnetic and unique at $2 / 3$ filling. The spin-spin correlation functions is fluctuating, non-vanishing eventually, with increasing the spatial distances, suggesting that the ground state has antiferromagnetic long-range order. The spin-charge correlation function is also fluctuating, implying that the spin and charge degrees of freedom are highly correlated in the state. The spin and charge are not seperated. The behaviors are quite different from those of Ising model.

The studies on the antiferromagnetic $t-J_{z}-V$ model with proper parameters at halffilling show that the system is very similar to the Ising model, as the spin-spin correlation function is exactly the same. The ground state is unique and has antiferromagnetic longrange order.

\section{ACKNOWLEDGMENTS}

The author is indebted to Dr. A. Klümper and Dr. A. Schadschneider for drawing his attention to this field, helpful discussions and advice. He is also grateful to Prof. J. Zittartz and ITP of Universität zu Köln for warm hospitality. This work is supported by the Alexander von Humboldt Stiftung. 


\section{REFERENCES}

* On leave from Graduate School, Chinese Academy of Sciences, Beijing, China. E-mail: gs@thp.uni-koeln.de.

[1] R.J. Baxter, Exactly Solved Models in Statistical Mechanics, (Academic Press, London, 1982).

[2] A. Klümper, A. Schadschneider, and J. Zittartz, J. Phys. A24, L955(1991); Z. Phys. B87, 281(1992); and Europhys. Lett. 24, 293(1993).

[3] J. de Boer and A. Schadschneider, Phys. Rev. Lett. 75, 4298(1995).

[4] For a review, see E. Dagotto and T.M. Rice, preprint cond-mat/9509181.

[5] E. Dagotto, J. Riera and D.J. Scalapino, Phys. Rev. B45, 5744(1992).

[6] S.P. Strong and A.J. Millis, Phys. Rev. Lett. 69, 2419(1992).

[7] T. Barnes et al., Phys. Rev. B47, 3196(1993).

[8] T.M.Rice, S. Gopalan and M. Sigrist, Europhys. Lett.23, 445(1993); S. Gopalan, T.M. Rice and M. Sigrist, Phys. Rev. B49, 8901(1994); M. Sigrist, T.M. Rice, and F.C. Zhang, Phys. Rev. B49, 12059(1994).

[9] M. Troyer, H. Tsunetsugu and D. Würtz, Phys. Rev. B50, 13515(1994).

[10] S.R. White, preprint cond-mat/9503104.

[11] D.G. Shelton, A.A. Nersesyan and A.M. Tsvelik, preprint cond-mat/9508047.

[12] Y. Xian, Phys. Rev. B52, 12485(1995).

[13] S. Takada and H. Watanabe, J. Phys. Soc. Jpn. 61, 39(1992).

[14] H. Watanabe, K. Nomura and S. Takada, J. Phys. Soc. Jpn. 62, 2845(1992).

[15] F.D.M. Haldane, Phys. Lett. 93A, 464(1983). 
[16] M. Takano, Z. Hiroi, M. Azuma and Y. Takeda, Jpn. J. Appl. Phys. 7, 3(1992).

[17] R.S. Eccleston, T. Barnes, J. Brody and J.W. Johnson, preprint cond-mat/9406010; T. Barnes, and J. Riera, preprint cond-mat/9404060.

[18] N.D. Mermin and H. Wagner, Phys. Rev. Lett. 17, 1133(1966).

[19] P. Schlottman, Phys. Rev. B36, 5177(1987).

[20] S. Kivelson, V. Emery and H.Q. Lin, Phys. Rev. B42, 6523(1990).

[21] E. Dagotto and J. Riera, Phys. Rev. B46, 12084(1992).

[22] M. Troyer, H. Tsunetsugu, T.M. Rice et al., Phys. Rev. B48, 4002(1993).

[23] E. Ising, Z. Physik, 31, 253(1925). 